\title{
The Wheelchair Use Confidence Scale: Italian translation, adaptation, and validation of the short form
}

Anna Berardi, Rita De Santis, Marco Tofani, Marquez Maru, Valter Santilli, Paula W. Rushton, Roberta Mollica \& Giovanni Galeoto

To cite this article: Anna Berardi, Rita De Santis, Marco Tofani, Marquez Maru, Valter Santilli, Paula W. Rushton, Roberta Mollica \& Giovanni Galeoto (2017): The Wheelchair Use Confidence Scale: Italian translation, adaptation, and validation of the short form, Disability and Rehabilitation: Assistive Technology, DOI: 10.1080/17483107.2017.1357053

To link to this article: http://dx.doi.org/10.1080/17483107.2017.1357053

View supplementary material ¿

Published online: 31 Jul 2017.

Submit your article to this journal $₫$

View related articles \ulcorner

View Crossmark data $\asymp$ 


\title{
The Wheelchair Use Confidence Scale: Italian translation, adaptation, and validation of the short form
}

\author{
Anna Berardi ${ }^{a}$, Rita De Santis ${ }^{a}$, Marco Tofani ${ }^{b}$, Marquez Maru ${ }^{c}$, Valter Santilli ${ }^{a}$, Paula W. Rushton ${ }^{d}$, Roberta Mollica ${ }^{a}$ \\ and Giovanni Galeoto ${ }^{\mathrm{e}}$ \\ ${ }^{a}$ Department of Anatomical, Histological, Forensic and Orthopaedic Sciences, Sapienza University of Rome, Rome, Italy; ${ }^{b}$ Department of \\ Neurosciences and Neurorehabilitation, Bambino Gesù Children's Hospital, Rome, Italy; 'Occupational Therapist of Paraplegic Center Ostia, Ostia, \\ Italy; ${ }^{d}$ Université de Montréal, Québec, Canada; CHU Sainte-Justine Research Center, Montréal, Québec, Canada; ${ }^{e}$ Department of Public Health, \\ Sapienza University of Rome, Rome, Italy
}

\begin{abstract}
Objective: We developed an Italian version of the Wheelchair Use Confidence Scale for Manual UsersShort Form (WheelCon-M-I-short form) and examined its reliability and validity.

Methods: The original scale was translated from English to Italian using the "Translation and Cultural Adaptation of Patient Reported Outcomes Measures-Principles of Good Practice" guidelines. The WheelCon-M-I-short form was administered to experienced manual wheelchair users who had a variety of diagnoses. Its internal consistency and test-retest reliability were examined. Its concurrent validity was evaluated using Pearson correlation coefficients with the Italian version of the Wheelchair Outcome Measure (WhOM-I) and the Italian version of the Barthel index (BI).

Results: The WheelCon-M-I-short form was administered to 31 subjects. The mean \pm SD of the WheelCon$\mathrm{M}$-I-short form score was 7.5 \pm 1.9 . All WheelCon-M-I-short form items were either identical or similar in meaning to the WheelCon-M-short form items. Cronbach's $\alpha$ for the WheelCon-M-I-short form was 0.95 $(p<0.01)$, and the test-retest reliability (ICC) was $0.978(p<0.01)$. The Pearson correlation coefficient of the WheelCon-M-I-short form scores with the WhOM-I scores was $0.7618(p<0.01)$. The Pearson correlation coefficient of the WheelCon-M-I-short form scores with the Italian BI scores was $0.638(p<0.01)$.

Conclusions: The WheelCon-M-I-short form was found to be reliable and a valid outcome measure for assessing manual wheelchair confidence in the Italian population.
\end{abstract}

> IMPLICATION FOR REHABILITATION

- The WheelCon-M-I-short form is a valid outcome measure available for assessing wheelchair confidence, according to Bandura's social cognitive theory, self-efficacy is a better predictor of future behavior than skill itself.

- Translation of the WheelCon-M-short form into the WheelCon-M-I-short form provides a new tool for Italian professionals.

- Clinicians now have a method to measure this invisible barrier to wheelchair use, and they will be able to make informed decisions when prescribing the use of manual wheelchairs and when training clients in their use.

- The WheelCon-M-I-short form also provides researchers with a tool in an important and relevant area of study for future research.
ARTICLE HISTORY

Received 13 April 2017

Accepted 15 July 2017

\section{KEYWORDS}

Self-efficacy; the Wheelchair Use Confidence Scale (WheelCon-M); validation; cultural adaptation; rehabilitation

\section{Introduction}

In Italy, about 13 million of people have functional limitations, disability, or chronic severe diseases, representing $25.5 \%$ of the total resident population [1]. Worldwide, the wheelchair plays a critical role in enhancing mobility, with approximately 65 million people requiring one [2]. In fact, the wheelchair is one of the most commonly used assistive technology devices for enhancing personal mobility, a precondition for those limited in mobility to enjoy human rights, live in dignity, and become more productive members within their own communities. For many people, an appropriate, well-designed, and well-fitted wheelchair can be the first step toward inclusion and social participation [1]. Over half of wheelchair users require assistance with using their wheelchair and with the activities of daily living [3]. The literature exploring factors affecting wheelchair use is mainly composed of variables related to the environment and the physical attributes of the wheelchair users [4,5], and a number of instruments have been developed to assess wheelchair skill in overcoming environmental barriers [6,7]. These predictive models only account for a modest amount of the variance, however [8].

Low self-efficacy has recently been recognized as an invisible barrier to manual wheelchair use [8]. According to Bandura's Social Cognitive Theory [9], self-efficacy is a better predictor of future behaviour than skill itself [10]. Self-efficacy has been

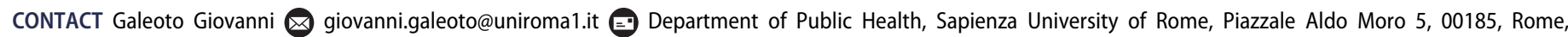
Italy

(4) Supplemental data for this article can be accessed here.

(C) 2017 Informa UK Limited, trading as Taylor \& Francis Group 
identified in a variety of rehabilitation areas, such as arthritis $[11,12]$, cardiac disease [13,14], end-stage renal disease [15], cancer $[16,17]$, exercise $[18,19]$, and anxiety [20] as an important factor in better outcomes.

Recently, a new self-report outcome measure was developed to assess manual wheelchair confidence. The difference between instruments that assess function or skill and this new scale is that the Wheelchair Use Confidence Scale for Manual users (WheelCon$\mathrm{M})$ is designed to ask about belief in one's ability to overcome aspects of the physical environment. It has been reported as reliable and valid when used with community-dwelling manual wheelchair users [21].

The Wheelchair Use Confidence Scale for Manual users-short form (WheelCon-M-short form) is a 21-item self-report questionnaire designed to measure wheelchair confidence in relation to two areas: managing the physical environment (13 items) and managing the social environment (eight items). Each item is scored using a 10-point Likert scale ranging from " 0 " (not confident) to "10" (completely confident) [22]. It is a reliable and valid instrument when used with community-dwelling manual wheelchair users [22,23], and with older adult manual wheelchair users $[24,25]$. At present, the WheelCon-M-short form is only available in English and French.

Therefore, the purpose of this study is to translate and culturally adapt the WheelCon-M-short form into Italian and examine its reliability and validity in a sample of wheelchair users.

\section{Methods}

After receiving the consent of the developers of the original instrument, the WheelCon-M-short form was translated from English to Italian using the "Translation and Cultural Adaptation of Patient Reported Outcomes Measures-Principles of Good Practice" guidelines [26].

\section{Translation and cultural adaptation}

The first stage in the adaptation was forward translation. The original Canadian version of the WheelCon-M-short form was translated into Italian by a panel of two native English speakers and one Italian clinical psychologist familiar with English. These individuals produced three independent translations. An independent native speaker of the target language who had not been involved in any of the forward translations synthesized the results of the translations. Working from the temporary version of the questionnaire, translated literally, three Italian translators then translated the questionnaire back into the original language without having seen the original version. The back-translated version of the instrument was compared with the original. This version has been submitted to the developers of the instrument and approved by them.

In order to adapt the translated version to Italian culture, two Italian rehabilitation professionals (an occupational therapist and a physiotherapist) and one clinical psychologist, who were familiar with both English and Italian, reviewed the first translated version and then reworded and reformulated some items to minimize any differences from the original version. The expert committee's role is to consolidate all the versions of the questionnaire and to develop what would be considered the pre-final version of the questionnaire for field testing.

\section{Pre-test (cross-cultural validity)}

This pre-final translated version of WheelCon-M-I-short form was preliminarily applied to 20 patients to evaluate its cross-cultural validity. In order to avoid bias, each patient was tested twice by the same professional. It is recommended that the time interval between repeated administrations be short enough to ensure that no clinical change had occurred. A time period of four to six days has been considered appropriate. On the basis of the results obtained from the preliminary application and analysis, no items were modified to improve comprehensibility and applicability. This resulted in the final Italian version of the WheelCon-M-short form being applied to the whole population of the study.

\section{Subjects}

According to the Canadian-French validation of the tool [27], a sample size of 29 subjects was required in order to achieve a level of reliability with a Cronbach's $\alpha>0.70$ and a 95\% confidence interval of $>0.70$ [28]. To be included in the study, participants had to be at least 18 years old, use a manual wheelchair as their primary means of mobility (at least $4 \mathrm{~h}$ per day), have at least six months of manual wheelchair experience, and communicate in Italian as their primary language. Individuals with emotional or psychiatric problems (as determined by clinical screening) were excluded from the study. Recruited participants who met the study inclusion criteria were scheduled for two testing sessions. The WheelCon-M-I-short form was administered at both baseline and follow-up by a trained ratter, an occupational therapist.

\section{Reliability}

The internal consistency of the WheelCon-M-I-short form was examined by Cronbach's $\alpha$ in order to assess the interrelatedness of the items and the homogeneity of the scale, Nunnally [29] has suggested that if a new questionnaire is to be used, its $\alpha$ coefficient should be at least 0.7 .

In order to evaluate the test-retest reliability, the WheelCon-MI-short form was administered twice to the population by the same professional. The time interval for test-retest studies needs to be sufficiently short to support the assumption that the patients remain stable and sufficiently long to prevent recall. According with the Canadian validation of the tool, a time interval of 7-14 days is considered appropriate for the current population. In order to measure test-retest reliability, the intraclass correlation coefficient (ICC) was calculated. An ICC value of $\geq 0.70$ is considered optimal to establish the degree to which repeated measurements are free from measurement error, so the scale was considered as stable at the test-retest for ICC of $>0.70$.

\section{Validity}

To evaluate concurrent validity, the WheelCon-M-I-short form, the wheelchair outcome measure-I (WhOm-I), and the Italian version of the Barthel index (BI) [30] were administered together and the Pearson correlation coefficients with the WheelCon-M-I-short form were calculated. All statistical analyses were done using IBM-SPSS version 23.00 (Armonk, NY).

\section{Results}

Participants were recruited from 31 January 2017, through the "Centro Paraplegici" of Ostia and the "Istituto di Ricovero e Cura a Carattere Scientifico Santa Lucia" of Rome. The WheelCon-M-Ishort form was administered to 31 adults along with other measures. The WhOM and the $\mathrm{BI}$ were also distributed to a subgroup $(n=20)$ of this sample. 
Table 1. Pre-test analysis.

\begin{tabular}{lc}
\hline & Sample $=20$ \\
\hline Age (mean \pm SD) & $52.25 \pm 15.26$ \\
Gender men $n(\%)$ & $13(65)$ \\
Diagnosis $n(\%)$ & \\
$\quad$ Quadriplegia & $4(20)$ \\
$\quad$ Paraplegia & $16(80)$ \\
WheelCon score (mean \pm SD) test & $8.35 \pm 1.11$ \\
WheelCon score (mean \pm SD) retest & $8.43 \pm 1.18$ \\
\hline
\end{tabular}

\section{Translation and cultural adaptation}

After forward and backward translation, approved by the developers of the tool on 16 January 2017, and after a consensus meeting, the translated scale was formed. The experts agreed that the example "For example, a person may be $8 \%$ confident they can memorize a grocery list of five items, but only $6 \%$ confident they can memorize a grocery list with 10 items" was misleading and asked for its deletion from the instructions section of the tool; the phrasing of some of the items was also reformulated. In addition, modifications were made to individual items with reference to the experts' opinions (see Supplementary Appendix 1).

\section{Pre-test (cross-cultural validity)}

Cross-cultural validity was evaluated on 20 subjects in January 2017. The characteristics of the subjects are summarized in Table 1. The results were strikingly similar to those found using the Canadian version, and no items were modified to improve comprehensibility and applicability (see Supplementary Appendix 2).

\section{Subjects}

From 7 February 7 2017, to 6 March 2017, 31 subjects who met the inclusion criteria were enrolled in the present study. The demographic characteristics of the subjects are summarized in Table 2. All patients were informed about the study, and their interest in taking part in it was recorded; those who entered the study gave their consent before inclusion [31,32].

\section{Reliability}

The WheelCon-M-I-short form was found to have an excellent degree of internal consistency. Cronbach's $\alpha$ for the WheelCon-MI-short form was $0.95(p<0.01)$. Internal consistency was calculated for the entire subscale; the item-item correlation and item-total correlation showed positive and statistically significant results, as reported in Table 3.

All 31 subjects were submitted to test-retest reliability procedures. The WheelCon-M-I-short form was reliable with respect to test-retest with an ICC of $0.978(p<0.01)$. The item-total correlations were significant $(p<0.01)$ and high for all questions. The test-retest reliability of each item is reported in Table 4.

\section{Validity}

The Pearson correlation coefficients of the Wheel-Con-M-I-short form with WhOM-I and the Italian $\mathrm{BI}[30]$ were $0.7618(p<0.01)$ and $0.638(p<0.01)$, respectively, indicating that the WheelCon-MI-short form has good concurrent validity. The Pearson correlation coefficient of each item is reported in Table 5.

\section{Discussion}

This study attempted to develop an Italian version of the WheelCon-M-short form and to evaluate its reliability and validity.
Table 2. Demographic characteristics of the subjects.

\begin{tabular}{|c|c|}
\hline & Sample $=31$ \\
\hline Age (mean $\pm S D)$ & $53.32 \pm 17.86$ \\
\hline Gender men $n(\%)$ & $18(58.1)$ \\
\hline Years using manual wheelchair (mean $\pm S D$ ) & $16.15 \pm 17.86$ \\
\hline Hours on WheelChair (mean $\pm S D$ ) & $15.52 \pm 2.14$ \\
\hline Months of rehabilitation (mean $\pm \mathrm{SD}$ ) & $11.29 \pm 9.49$ \\
\hline Months of occupational therapy (mean \pm SD) & $7.68 \pm 6.85$ \\
\hline \multicolumn{2}{|l|}{ Diagnosis $n(\%)$} \\
\hline Hemiplegia & $6(19.4)$ \\
\hline Paraplegia & $17(54.8)$ \\
\hline infantile cerebral palsy & $1(3.2)$ \\
\hline Multiple sclerosis & $2(6.4)$ \\
\hline Quadriplegia & $4(12.9)$ \\
\hline Head trauma & $1(3.2)$ \\
\hline \multicolumn{2}{|l|}{ Marital status (\%) } \\
\hline Single & $10(32.3)$ \\
\hline Married/common-law & $15(48.4)$ \\
\hline Divorced & $1(3.2)$ \\
\hline Widowed & $5(16.1)$ \\
\hline \multicolumn{2}{|l|}{ Education $n$ (\%) } \\
\hline High school & $21(67.7)$ \\
\hline University & $10(32.3)$ \\
\hline \multicolumn{2}{|l|}{ Living environment $n$ (\%) } \\
\hline Rural & $6(19.4)$ \\
\hline City & $17(54.8)$ \\
\hline Suburban & $8(25.8)$ \\
\hline \multicolumn{2}{|l|}{ Lives $n(\%)$} \\
\hline With spouse/children/parents & $27(87.1)$ \\
\hline Alone & $4(12.9)$ \\
\hline \multicolumn{2}{|l|}{ Employment status $n$ (\%) } \\
\hline Fulltime work at home & $1(3.2)$ \\
\hline Unemployed & $5(16.1)$ \\
\hline Employed & $5(16.1)$ \\
\hline Retired & $15(48.4)$ \\
\hline Student & $1(3.2)$ \\
\hline Volunteer & $4(12.9)$ \\
\hline \multicolumn{2}{|l|}{ Method of propulsion $n$ (\%) } \\
\hline Two hands & $26(83.9)$ \\
\hline One hand & $4(12.9)$ \\
\hline One hand, one foot & $1(3.2)$ \\
\hline
\end{tabular}

In this article, we have reported on the translation, the cultural adaptation of the short form of the WheelCon-M for use among Italian wheelchair users, and the subsequent evaluation of its validity evidence.

Translation and linguistic adaptation were performed as for the Canadian-French version. Application of the "Translation and cultural adaptation of patient reported outcomes measures-principles of good practice" guidelines [26] proved to be straightforward under the supervision of the developers of the instrument and a panel of experts who ensured the maintenance of the original meaning of the items.

Participants in this study were experienced, community-dwelling adults ranging in age from 27 to 79, with a variety of diagnoses. Generally, they were quite confident with wheelchair use; the mean WheelCon-M-I-short form in this study $(7.5 \pm 1.9)$ was quite similar to that of the Canadian-French version of the long form (WheelCon-M-F) $(63.8 \pm 19.9)$ and to that obtained by Rushton et al. in their WheelCon-M-long form measurement study (84.6, interquartile range 71.3-92.0).

It was determined that the WheelCon-M-I-short form has high internal consistency and strong retest reliability. It also showed significant positive correlations with other measures, including the WhOM-I and the Italian validated version of the $\mathrm{BI}$ [30], thus confirming its validity.

The most important index of test reliability is the $\alpha$ coefficient [33]. The WheelCon-M-I-short form produced similar results to the WheelCon-M-F-long form and the WheelCon-M-long form; all 
Table 3. Item-tot item analysis.

\begin{tabular}{|c|c|c|c|c|c|}
\hline & $\begin{array}{l}\text { Scale mean if } \\
\text { item deleted }\end{array}$ & $\begin{array}{l}\text { Scale variance } \\
\text { if item deleted }\end{array}$ & $\begin{array}{c}\text { Corrected item-total } \\
\text { correlation }\end{array}$ & $\begin{array}{c}\text { Squared multiple } \\
\text { correlation }\end{array}$ & $\begin{array}{l}\text { Cronbach's } \alpha \text { if } \\
\text { item deleted }\end{array}$ \\
\hline Item 1 & 142.1 & 1663.624 & 0.445 & 0.945 & 0.95 \\
\hline Item 2 & 141.84 & 1664.606 & 0.517 & 0.972 & 0.95 \\
\hline Item 3 & 141.65 & 1634.103 & 0.516 & 0.929 & 0.949 \\
\hline Item 4 & 144.13 & 1547.983 & 0.563 & 0.91 & 0.95 \\
\hline Item 5 & 144.84 & 1464.606 & 0.811 & 0.962 & 0.945 \\
\hline Item 6 & 142.29 & 1587.68 & 0.72 & 0.936 & 0.947 \\
\hline Item 7 & 145.35 & 1469.237 & 0.809 & 0.98 & 0.945 \\
\hline Item 8 & 143.45 & 1496.323 & 0.83 & 0.944 & 0.945 \\
\hline Item 9 & 143.87 & 1568.983 & 0.753 & 0.915 & 0.946 \\
\hline Item 10 & 144.77 & 1457.914 & 0.847 & 0.976 & 0.945 \\
\hline Item 11 & 145.87 & 1481.049 & 0.827 & 0.978 & 0.945 \\
\hline Item 12 & 147.1 & 1520.224 & 0.776 & 0.977 & 0.946 \\
\hline Item 13 & 147.35 & 1525.37 & 0.739 & 0.982 & 0.946 \\
\hline Item 14 & 142.1 & 1606.957 & 0.586 & 0.974 & 0.949 \\
\hline Item 15 & 141.97 & 1612.566 & 0.549 & 0.981 & 0.949 \\
\hline Item 16 & 142.03 & 1575.699 & 0.814 & 0.951 & 0.946 \\
\hline Item 17 & 142.23 & 1555.447 & 0.707 & 0.975 & 0.947 \\
\hline Item 18 & 143.16 & 1544.806 & 0.756 & 0.943 & 0.946 \\
\hline Item 19 & 141.61 & 1654.312 & 0.447 & 0.877 & 0.95 \\
\hline Item 20 & 141.45 & 1640.923 & 0.61 & 0.96 & 0.949 \\
\hline Item 21 & 142.45 & 1628.789 & 0.618 & 0.918 & 0.948 \\
\hline
\end{tabular}

Table 4. Test-retest analysis.

\begin{tabular}{lccc}
\hline & Test & Retest & ICC IC 95\% \\
\hline Item 1 & $8.48 \pm 1.57$ & $8.84 \pm 1.37$ & $0.951[0.831 ; 0.958]$ \\
Item 2 & $8.74 \pm 1.34$ & $9.03 \pm 2$ & $0.920[0.841 ; 0.961]$ \\
Item 3 & $8.94 \pm 2.03$ & $9.03 \pm 2.04$ & $0.973[0.945 ; 0.987]$ \\
Item 4 & $6.45 \pm 3.65$ & $6.52 \pm 3.81$ & $0.988[0.976 ; 0.994]$ \\
Item 5 & $5.74 \pm 3.92$ & $5.81 \pm 3.94$ & $0.987[0.974 ; 0.994]$ \\
Item 6 & $8.29 \pm 2.27$ & $8.26 \pm 2.27$ & $0.977[0.954 ; 0.989]$ \\
Item 7 & $5.23 \pm 3.85$ & $5.35 \pm 3.73$ & $0.975[0.949 ; 0.988]$ \\
Item 8 & $7.13 \pm 3.35$ & $7.23 \pm 3.31$ & $0.987[0.973 ; 0.994]$ \\
Item 9 & $6.71 \pm 2.47$ & $6.87 \pm 2.4$ & $0.955[0.908 ; 0.978]$ \\
Item 10 & $5.81 \pm 3.86$ & $5.87 \pm 3.94$ & $0.989[0.978 ; 0.995]$ \\
Item 11 & $4.71 \pm 3.59$ & $4.94 \pm 3.79$ & $0.964[0.927 ; 0.983]$ \\
Item 12 & $3.48 \pm 3.18$ & $3.48 \pm 3.27$ & $0.754[0.549 ; 0.873]$ \\
Item 13 & $3.23 \pm 3.24$ & $3.32 \pm 3.35$ & $0.839[0.693 ; 0.919]$ \\
Tot & $82.94 \pm 30.92$ & $84.55 \pm 31.08$ & $0.974[0.946 ; 0.987]$ \\
Item 14 & $8.48 \pm 2.35$ & $8.61 \pm 2.2$ & $0.982[0.964 ; 0.991]$ \\
Item 15 & $8.61 \pm 2.38$ & $8.68 \pm 2.56$ & $0.962[0.923 ; 0.982]$ \\
Item 16 & $8.55 \pm 2.2$ & $8.58 \pm 2.14$ & $0.982[0.964 ; 0.992]$ \\
Item 17 & $8.35 \pm 2.86$ & $8.26 \pm 2.83$ & $0.990[0.980 ; 0.995]$ \\
Item 18 & $7.42 \pm 2.86$ & $7.48 \pm 2.87$ & $0.972[0.943 ; 0.986]$ \\
Item 19 & $8.97 \pm 1.8$ & $8.90 \pm 1.87$ & $0.991[0.981 ; 0.996]$ \\
Item 20 & $9.13 \pm 1.6$ & $9.13 \pm 1.61$ & 1 \\
Item 21 & $8.13 \pm 1.82$ & $8.39 \pm 1.78$ & $0.928[0.857 ; 0.965]$ \\
Tot & $67.65 \pm 15.45$ & $68.03 \pm 15.36$ & $0.979[0.957 ; 0.990]$ \\
Tot WheelCon & $150.58 \pm 41.51$ & $152.58 \pm 41.58$ & $0.978[0.954 ; 0.989]$ \\
\hline
\end{tabular}

Table 5 Gold standard analysis.

\begin{tabular}{lccc}
\hline & Fiscal test & Social test & WheelCon \\
\hline WhOMnl part & $0.617^{\mathrm{b}}$ & $0.520^{\mathrm{a}}$ & $0.718^{\mathrm{b}}$ \\
WhOMIl part & $0.759^{\mathrm{b}}$ & 0.435 & $0.796^{\mathrm{b}}$ \\
Barthel & $0.629^{\mathrm{b}}$ & 0.308 & $0.638^{\mathrm{b}}$ \\
\hline
\end{tabular}

${ }^{a}$ Correlation is significant at the level of 0.05 (two-tailed).

${ }^{\mathrm{b}}$ Correlation is significant at the level of 0.01 (two-tailed).

demonstrated high internal consistency, with a Cronbach's $\alpha$ of $0.95,0.98$, and 0.92 , respectively.

The WheelCon-M-I-short form ICC of 0.978 for retest reliability was very good. The high level of interrelatedness among the items shows the cross-cultural validity of the adapted scale, which reflects the performance of the original Canadian version [34]. Furthermore, all versions produced excellent test-retest reliability, with ICCs of $0.978,0.87$, and 0.84 for the Italian translation of the short form, French-Canadian translation, and original WheelCon-M long form, respectively. The high WheelCon-M-Ishort form reliability indicates that the scores of the patients remain stable after repeated measurement, as in the original version.

The Pearson correlation coefficient of the Wheel-Con-M-I-short form with the other measures indicates good concurrent validity. Consistent with the original research, we used an experienced sample of participants to increase the likelihood of stability of confidence during the time between tests. Our results corroborate most of the a priori hypotheses regarding the relationships between the variables used to assess concurrent validity and the WheelCon-M-I-short form. The magnitudes of the relationships between the WheelCon-M-I-short form and the WhOM-I and Italian BI were as expected, given that approximately half of the WheelCon-M-short form items involved "managing physical environment," the focus of the WhOM and BI. The other WheelCon-M-I-short form items involved assessing confidence related to activities, knowledge and problem solving, advocacy, managing social situations, and managing emotions, areas not covered in the WhOM and $\mathrm{BI}$ [35].

Because situation-specific self-efficacy measures provide more explanatory and predictive value than do global measures of selfefficacy [36], the mobility and self-management subscales may provide self-efficacy estimates that are more accurate for certain clinical situations or research questions than the 21-item WheelCon-M-short form does. For example, for outcomes related to mobility or physical abilities, the use of the 13-item mobility efficacy subscale may be more predictive than the eight-item selfmanagement efficacy subscale. Similarly, the reverse may be true for outcomes related to problem solving, advocacy, or other selfmanagement tasks. Although the 21-item short form does not function as well as the individual subscales, as indicated by the misfitting items, it may still be of value, particularly for the prediction of multifaceted outcomes, such as participation in social and personal roles or other life situations. Research investigating this hypothesis is needed. Offering reliable and valid situation-specific measurements along with a more global measurement may facilitate the assessment of wheelchair use self-efficacy both clinically and in research [22]. 


\section{Limitations of the study}

This study has a number of limitations. A major limitation is the sample size; some may consider a sample of 31 to be small, and it is not large enough to examine the influences of the various sociodemographic variables. Even if it was less than that required, however, the precision of our confidence interval was equal to that of the original Canadian and French-Canadian long versions of WheelCon.

In using WheelCon-M-I-short form, however, it is important to recognize that the reliability and validity described above are limited to a sample of older wheelchair users who had at least six months of experience in manual wheelchair use. It is also important to note that the sample had a higher than average percentage of spinal cord injury diagnosis (74\%). Although a more stable sample was necessary to evaluate the retest reliability of the WheelCon-M-I-short form, this limited the generalizability of the results. For instance, it would be interesting to test the measurement properties with new wheelchair users or diagnosis-specific samples and to validate other versions of the WheelCon (for powered wheelchair users, for children, and for caregivers).

Furthermore, the sample was restricted to the context of Rome, which is a limitation in that culturally diverse perspectives were absent. Finally, despite having a small rural representation included in the sample, the impact of different geographies and climates on wheelchair use outside of Rome was also missing. Although further work is needed, this new test holds promise as a clinical and research tool [10].

\section{Conclusions}

In conclusion, the culturally adapted WheelCon-M-I-short form shows itself to be a valid, reliable, acceptable, easy-to-understand, and rapidly administrable scale to measure confidence with wheelchair use in the Italian population; it was not available prior to this work. This work provides a new tool for Italian professionals to measure and capture wheelchair confidence data across Italy.

Despite the study's limitations and the need for further study, the WheelCon-M-I-short form is the first measure of confidence with manual wheelchair use to have been rigorously developed and psychometrically tested in the Italian context. Results obtained in this study provide data on the measurement properties of this new tool [36].

Clinicians now have a method to measure this invisible barrier to wheelchair use, and they will be able to make informed decisions when prescribing the use of manual wheelchairs and when training clients in their use. The WheelCon-M-I-short form also provides researchers with a tool in an important and relevant area of study for future research [21].

\section{Disclosure of statement}

The authors declare that they have no conflict of interest.

Statement of Human and Animal Rights: All procedures followed were in accordance with the ethical standards of the responsible committee on human experimentation (institutional and national) and with the Helsinki Declaration of 1975, as revised in 2008 (5). Informed consent was obtained from all patients for being included in the study.

Statement of informed Consent: Informed consent was obtained from all individual participants included in the study

\section{Funding}

All authors have no commercial associations or disclosures that may pose or create a conflict of interest with the information presented within this manuscript.

\section{References}

[1] Ministero del lavoro e delle politiche sociali Inclusione Sociale Delle Persone Con Limitazioni Funzionali, Invalidità O Cronicità Gravi Anno 2013 Nel 2013, circa 13 milioni. 25 luglio 2015 - ISTAT Available from: http://www.istat.it/it/ files/2015/07/Inclusione-sociale-persone-con-limitazioni-funzionali_def_240715.pdf?title=Limitazioni+funzioni $\% 2 C+$ invalidit $\%$ C3\%A0\%2Ccronicit\%C3\%A0+gravi+-+21\%2Flug\% 2F2015+-+Testo+integrale.pdf.

[2] World Health Organization. Guidelines on the provision of manual wheelchairs in less resourced settings. Geneva: WHO Press; 2008.

[3] Shields M. Use of wheelchairs and other mobility support devices. Health Rep. 2004;15:37-40.

[4] Mortenson W, Miller WC, Backman C, et al. Association between mobility, participation, and wheelchair-related factors in long-term care residents who use wheelchairs as their primary means of mobility. J Am Geriatr Soc. 2012;60:1310-1315.

[5] Bourbonniere MC, Fawcett IM, Miller WC, et al. Prevalence and predictors of need for seating intervention and mobility for persons in long-term care. Can J Aging. 2007;26: 195-204.

[6] Stanley RK, Stafford DJ, Rasch E, et al. Development of a functional assessment measure for manual wheelchair users. J Rehabil Res Dev. 2003;40:301-307.

[7] Kilkens OJ, Dallmeijer AJ, de Witte LP, et al. The wheelchair circuit: construct validity and responsiveness of a test to assess manual wheelchair mobility in persons with spinal cord injury. Arch Phys Med Rehabil. 2004;85:424-431.

[8] Rushton PW, Miller WC. Development of an assessment to measure self-efficacy with wheelchair mobility [abstract]; CJOT. 2009. p. 76. Online supplement. Available from: http://www.caot.ca/default.asp?pageid $=2330$.

[9] Bandura A. Self-efficacy: the exercise of control. New York: W. H. Freeman and Company; 1997.

[10] Rushton P, Miller WC. Wheelchair Use Confidence Scale (WheelCon). February 25, 2013.

[11] Allegrante JP, Kovar PA, MacKenzie CR, et al. A walking education program for patients with osteoarthritis of the knee: theory and intervention strategies. Health Educ Q. 1993;20:63-81.

[12] Lorig KR, Sobel DS, Stewart AL, et al. Evidence suggesting that a chronic disease self-management program can improve health status while reducing hospitalization: a randomized trial. Med Care. 1999;37:5-14.

[13] Allen JK. Coronary risk factor modification in women after coronary artery bypass surgery. Nurs Res. 1996;45:260.

[14] Moore SM, Charvat JM, Gordon NH, et al. Effects of a CHANGE intervention to increase exercise maintenance following cardiac events. Ann Behav Med. 2006;200631:53.

[15] Tsay S. Self-efficacy training for patients with end-stage renal disease. J Adv Nurs. 2003;43:370-375.

[16] Lev EL, Owen SV. Counseling women with breast cancer using principles developed by albert bandura. Perspect Psychiatr Care. 2000;36:131-138. 
[17] Lev EL, Daley $\mathrm{KM}$, Conner $\mathrm{NE}$, et al. An intervention to increase quality of life and self-care self-efficacy and decrease symptoms in breast cancer patients. Scholar Inq Nurs Pract. 2001;15:277-294.

[18] McAuley E, Courneya KS, Rudolph DL, et al. Enhancing exercise adherence in middle-aged males and females. Prev Med. 1994;23:498-506.

[19] Allison MJ, Keller C. Self-efficacy intervention effect on physical activity in older adults. West J Nurs Res. 2004; 26:31-58.

[20] Fisher PA, Laschinger HS. A relaxation training program to increase self-efficacy for anxiety control in alzheimer family caregivers. Holistic Nurs Pract. 2001;15:47-58.

[21] Rushton PW, Miller WC, Kirby RL, et al. Development and content validation of the wheelchair use confidence scale: a mixed-methods study. Disabil Rehabil Assist Technol. 2011;6:57-66.

[22] Sakakibara BM, Miller WC, Rushton PW. Rasch analyses of the wheelchair use confidence scale. Arch Phys Med Rehabil J. 2015;96:1036-1044. homepage: www.archivespmr.org Archives of Physical Medicine and Rehabilitation

[23] Sakakibara BM, Miller WC, Eng JJ, et al. Preliminary examination of the relation between participation and confidence in older manual wheelchair users. Arch Phys Med Rehabil. 2013;94:791-794.

[24] Sakakibara BM, Miller WC, Routhier F, et al. Association between self-efficacy and participation in community-dwelling manual wheelchair users aged 50 years or older. Phys Ther. 2014;94:664-674.

[25] Sakakibara BM, Miller WC, Souza M, et al. Wheelchair skills training to improve confidence with using a manual wheelchair among older adults: a pilot study. Arch Phys Med Rehabil. 2013;94:1031-1037.

[26] Wild D, Grove A, Martin M, ISPOR Task Force for Translation and Cultural Adaptation, et al. Principles of good practice for the translation and cultural adaptation process for patient-reported outcomes (PRO) measures: report of the ISPOR task force for translation and cultural adaptation. Value Health. 2005;8:94-104.

[27] Rushton PW1, Routhier F, Miller WC, et al. French-Canadian translation of the WheelCon-M (WheelCon-M-F) and evaluation of its validity evidence using telephone administration. Disabil Rehabil. 2015;37:812-819.

[28] Shoukri MM, Asyali MH, Donner A. Sample size requirements for the design of reliability study: review and new results. Stat Methods Med Res. 2004;13:251-271.

[29] Nunnally JC. Psychometric theory. New York: McGraw-Hill; 1978.

[30] Galeoto G, Lauta A, Palumbo A, et al. The Barthel index: Italian translation, adaptation and validation. Int J Neurol Neurother. 2015;2:2. ISSN: 2378-3001

[31] Galeoto G, De Santis R, Marcolini A, et al. The informed consent in occupational therapy: proposal of forms. Giornale Italiano Med Lavoro Ergonomia. 2016;38(2): 107-115.

[32] Galeoto G, Mollica R, Astorino O, et al. Informed consent in physiotherapy: proposal of a form. Giornale Italiano Med Lavoro Ergonomia 2015;37(4):245-254.

[33] Kline P. Test construction: factor analytic and item analytic methods; In: Kline P. Editor. Handbook of psychological testing, 2nd ed. London: Routledge; 2000; p. 161-181.

[34] Terwee CB, Bot SD, de Boer MR, et al. Quality criteria were proposed for measurement properties of health status questionnaires. J Clin Epidemiol. 2007;60:34-42. 30.

[35] Rushton PW, Miller WC, Kirby RL, Measure for the assessment of confidence with manual wheelchair use (WheelCon-m) version 2.1: reliability and validity. J Rehabil Med. 2012;45:61-67.

[36] Bandura A. Guide for creating self-efficacy scales. In: Pajares F, Urdan T, editors. Self-efficacy beliefs of adolescents. Greenwich: Information Age Publishing; 2006; p. 307-337. 